Article

\title{
Optimizing a Male Reproductive Aging Mouse Model by D-Galactose Injection
}

\author{
Chun-Hou Liao ${ }^{1,2,3,+}$, Bing-Huei Chen ${ }^{4,+}$, Han-Sun Chiang ${ }^{3,5,+}$, Chiu-Wei Chen ${ }^{5}$, \\ Mei-Feng Chen ${ }^{6}$, Chih-Chun Ke ${ }^{7}$, Ya-Yun Wang ${ }^{8}$, Wei-Ning Lin ${ }^{5}$, Chi-Chung Wang ${ }^{5}$ and \\ Ying-Hung Lin ${ }^{5, *}$
}

Received: 9 September 2015; Accepted: 28 December 2015; Published: 13 January 2016

Academic Editor: Kenji Murata

1 PhD Program in Nutrition \& Food Science, Fu Jen Catholic University, No. 510, Zhongzheng Road, Xinzhuang District, New Taipei City 242, Taiwan; liaoch22@gmail.com

2 Division of Urology, Department of Surgery, Cardinal Tien Hospital, No. 362, Zhongzheng Road, Xindian District, New Taipei City 231, Taiwan

3 School of Medicine, Fu Jen Catholic University, No. 510, Zhongzheng Road, Xinzhuang District, New Taipei City 242, Taiwan; 053824@mail.fju.edu.tw

4 Department of Food Science, Fu Jen Catholic University, No. 510, Zhongzheng Road, Xinzhuang District, New Taipei City 242, Taiwan; 002622@mail.fju.edu.tw

5 Graduate Institute of Basic Medicine, Fu Jen Catholic University, No. 510, Zhongzheng Road, Xinzhuang District, New Taipei City 242, Taiwan; okwapnice@hotmail.com (C.-W.C.); 081551@mail.fju.edu.tw (W.-N.L.); 075006@mail.fju.edu.tw (C.-C.W.)

6 Research Center for Emerging Viral Infections, Chang Gung University, No. 259 Wen-Hwa 1st Road, Kwei-Shan Taoyuan 333, Taiwan; mfchen0@gmail.com

7 Department of Urology, En Chu Kong Hospital, No. 399, Fuxing Road, Sanxia District, New Taipei City 237, Taiwan; koacurtis@gmail.com

8 Department of Chemistry, Fu Jen Catholic University, No. 510, Zhongzheng Road, Xinzhuang District, New Taipei City 242, Taiwan; vic0009@gmail.com

* Correspondence: 084952@mail.fju.edu.tw; Tel.: +886-2-2905-3399; Fax: +886-2-2905-3415

+ These authors contributed equally to this work.

\begin{abstract}
The D-galactose (D-gal)-injected animal model, which is typically established by administering consecutive subcutaneous D-gal injections to animals for approximately six or eight weeks, has been frequently used for aging research. In addition, this animal model has been demonstrated to accelerate aging in the brain, kidneys, liver and blood cells. However, studies on aging in male reproductive organs that have used this animal model remain few. Therefore, the current study aimed to optimize a model of male reproductive aging by administering D-gal injections to male mice and to determine the possible mechanism expediting senescence processes during spermatogenesis. In this study, C57Bl/6 mice were randomized into five groups (each containing 8-10 mice according to the daily intraperitoneal injection of vehicle control or 100 or $200 \mathrm{mg} / \mathrm{kg}$ dosages of D-gal for a period of six or eight weeks). First, mice subjected to D-gal injections for six or eight weeks demonstrated considerably decreased superoxide dismutase activity in the serum and testis lysates compared to those in the control group. The lipid peroxidation in testis also increased in the D-gal-injected groups. Furthermore, the D-gal-injected groups exhibited a decreased ratio of testis weight/body weight and sperm count compared to the control group. The percentages of both immotile sperm and abnormal sperm increased considerably in the D-gal-injected groups compared to those of the control group. To determine the genes influenced by the D-gal injection during murine spermatogenesis, a c-DNA microarray was conducted to compare testicular RNA samples between the treated groups and the control group. The D-gal-injected groups exhibited RNA transcripts of nine spermatogenesis-related genes (Cycl2, Hk1, Pltp, Utp3, Cabyr, Zpbp2, Speer2, Csnka2ip and Katnb1) that were up- or down-regulated by at least two-fold compared to the control group. Several of these genes are critical for forming sperm-head morphologies or maintaining
\end{abstract}


nuclear integration (e.g., cylicin, basic protein of sperm head cytoskeleton 2 (Cylc2), casein kinase 2, alpha prime interacting protein (Csnka2ip) and katanin p80 (WD40-containing) subunit B1 (Katnb1)). These results indicate that D-gal-injected mice are suitable for investigating male reproductive aging.

Keywords: aging; male infertility; mouse model

\section{Introduction}

\subsection{Aging and Male Infertility}

The increasing trend of delayed parenthood in developed countries is accompanied by an increase in couples with reduced fertility [1,2]. In approximately $50 \%$ of couples with reduced fertility, fertility defects can be traced to male partners [3]. In the past decade, several large-scale clinical studies have indicated that male aging is accompanied by decreased semen quality (e.g., reduced total sperm count, concentration and motility), a decrease in the normal morphological sperm ratio and DNA-fragmented sperm [4,5]. In addition, the major reason for diminished semen quality is the accumulation of reactive oxygen species (ROS) and the loss of telomerase activity that accompanies aging [6,7].

\subsection{Reactive Oxygen Species (ROS) Generation in Testes}

The major roles of mitochondria include generating cellular energy and regulating apoptosis and other cellular processes [8]. Energy production through the electron transport chain is coupled with ROS production [8]. Antioxidant enzymes within mitochondria catalyze the ROS to hydro-oxygen and then to $\mathrm{H}_{2} \mathrm{O}$ [9]. However, mitochondrial dysfunction induces excessive ROS production and disrupts the mitochondrial antioxidant system [10]. ROS accumulation causes DNA damage, lipid peroxidation and malformed folding proteins. A clinical study reported that ROS levels were considerably higher in seminal ejaculates of healthy fertile males older than 40 years [6]. In addition, other studies have reported increased ROS levels in the pathogenesis of male infertility [11,12]. In addition, Desai et al. indicated that senescence processes in reproductive cells induced ROS accumulation in sperm mitochondria and Leydig cells, resulting in the disruption of sperm telomere, steroidogenesis in the Leydig cells and the presence of mitochondrial DNA in both cells [7].

\subsection{Effects of Oral Antioxidants on Male Infertility}

Several systematic reviews have indicated that oral antioxidants improve sperm quality (i.e., sperm count, motility and morphology) and pregnancy rates [13]. In addition, the use of oral-antioxidant-related products (e.g., vitamin C, vitamin E, selenium, folate, zine and carnitine) in treating men with infertility has been recommended [13,14]. However, individual studies investigating the therapeutic effect of such products have reported conflicting results $[15,16]$. Nevertheless, animal studies that have validated the effects of oral antioxidants on male reproductive aging are scant.

\subsection{Model of Male Reproductive Aging}

The most prevalent animal models used for investigating male reproductive aging involve using aging rats and D-galactose (D-gal)-injected rat and mouse models [17-20]. A previous study reported that aging male rats exhibited reduced serum testosterone levels because of decreased serum luteinizing hormone (LH) levels and repressed activates of antioxidant enzymes in Leydig cells $[17,18,21]$. Another study on aging rats revealed that genes involved in DNA induction damage and repair pathways were affected in the pachytene spermatocytes [22]. Furthermore, D-gal-induced brain aging models have been established for more than 15 years [20,23]. D-Gal is a reducing sugar normally existing in the body. When the concentration of D-gal exceeds normal levels, it is converted to aldehydes and $\mathrm{H}_{2} \mathrm{O}_{2}$ [20,24]. Animals injected with D-gal exhibit aging-related phenotypes, such as 
neurological defects, decreased immune responses, reduced antioxidant enzyme activity and increased ROS production $[20,23,25]$. However, studies on male reproductive aging in D-gal-injected mouse models have been reported only in recent years. Only Ahangarpour et al. used a D-gal-injected mouse model and reported decreased sperm counts and increased serum LH levels [19]. Nevertheless, details about D-gal-induced aging effects on semen parameters and possible molecular effects on male sperm cells remain unknown. Therefore, we optimized a model of male reproductive aging involving D-gal-injected mice to thoroughly evaluate the effects of D-gal on male reproductive aging.

\section{Results and Discussion}

\subsection{Optimizing a Model of Male Reproductive Aging through D-Galactose (D-Gal) Injection}

Previous studies on brain aging have indicated that the recommended D-gal dosage for optimizing an aging model is $50-200 \mathrm{mg} / \mathrm{kg}$ over a period of 1-2 months [20,23]. In the current study, to optimize a model of male reproductive aging, a total of 50 mice (aged eight weeks) were divided into five groups according to the administered D-gal dosage and injection period (Figure 1A): Group 1 (G1; a D-gal dosage of $100 \mathrm{mg} / \mathrm{kg}$ administered daily over six weeks), Group 2 (G2; a D-gal dosage of $100 \mathrm{mg} / \mathrm{kg}$ administered over eight weeks), Group 3 (G3; a D-gal dosage of $200 \mathrm{mg} / \mathrm{kg}$ administered over six weeks ), Group 4 (G4; a D-gal dosage of $200 \mathrm{mg} / \mathrm{kg}$ administered over eight weeks) and the vehicle group (control group; Phosphate-buffered saline (PBS) administered over eight weeks). The mice were sacrificed within $24 \mathrm{~h}$ after the final injection. To determine the effect of D-gal-induced aging, the superoxide dismutase (SOD) activity and the level of an antioxidant maker in the aging model were evaluated in the serum samples. The D-gal-injected groups exhibited significantly lower SOD activity compared to the control group (Figure 1B). In the testicular samples, SOD activity decreased slightly (Figure 2A); compared to the control group, only G1 demonstrated a significant decrease in SOD activity. To thoroughly evaluate the effect of D-gal injection on the aging of the testicular samples, malondialdehyde (MDA), a type of lipid peroxidation, was analyzed using a thiobarbituric acid reactive substances (TBARS) assay. The TBARS levels in the D-gal-injected mice (G1-G4) increased compared to that in the control group (Figure 2B). Among the experimental groups, G4 showed the highest TBARS level. According to the SOD and TBARS levels, antioxidant activity in the D-gal-injected mice decreased, inducing oxidative stress in testis. These results are similar to those obtained in the studies that evaluated brain aging in mouse models [20].

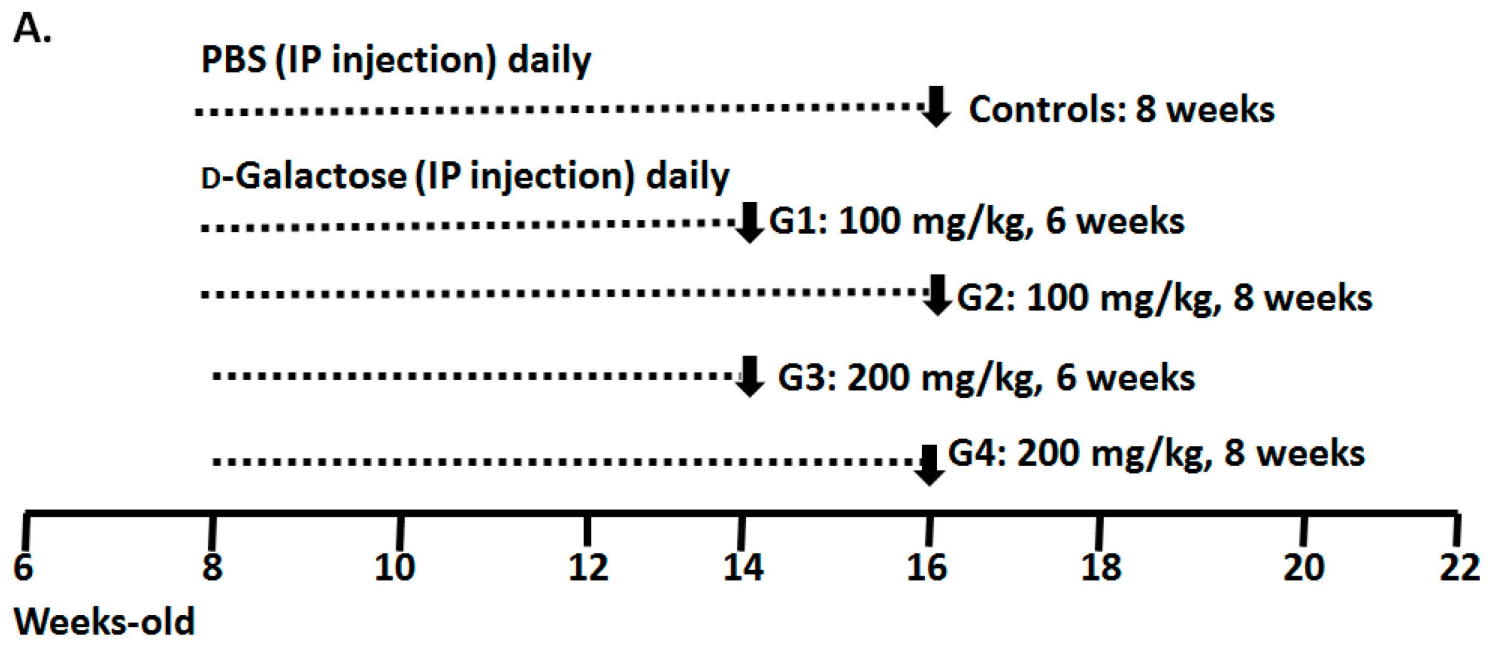

Figure 1. Cont. 
B.

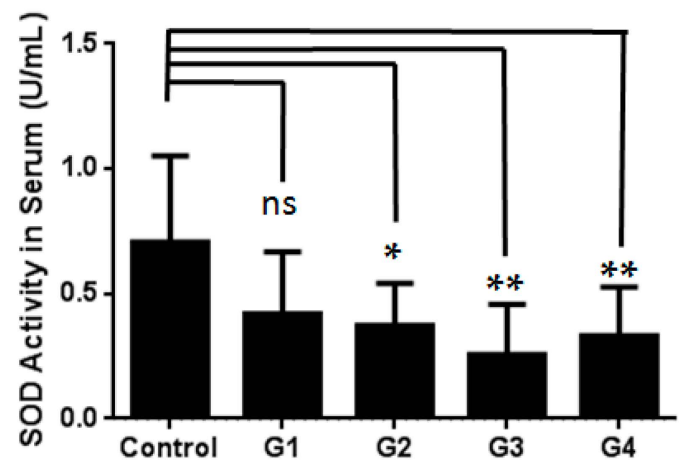

Figure 1. Model of male reproductive aging optimized through D-Galactose (D-Gal) injections. (A) Mice divided into five groups: the vehicle group (Control: Phosphate-buffered saline, PBS, administered over eight weeks) and the D-Gal-injected groups, namely G1 (D-Gal dosage of $100 \mathrm{mg} / \mathrm{kg}$ over six weeks), G2 (D-Gal dosage of $100 \mathrm{mg} / \mathrm{kg}$ over eight weeks), G3 (D-Gal dosage of $200 \mathrm{mg} / \mathrm{kg}$ over six weeks) and G4 (D-Gal dosage of $200 \mathrm{mg} / \mathrm{kg}$ over eight weeks). The mice were injected intraperitoneally daily for six or eight weeks. The bars indicate the ages of the mice in weeks; (B) The serum of mice injected with D-Gal showed lower superoxide dismutase) SOD activity (SOD is a critical antioxidant enzyme) than did the serum of those administered PBS. One-way Analysis of variance (ANOVA) test: "ns" denotes nonsignificant; bars represent the mean $\pm \mathrm{SD} ;{ }^{*} p<0.05 ;{ }^{* *} p<0.01$.

A.

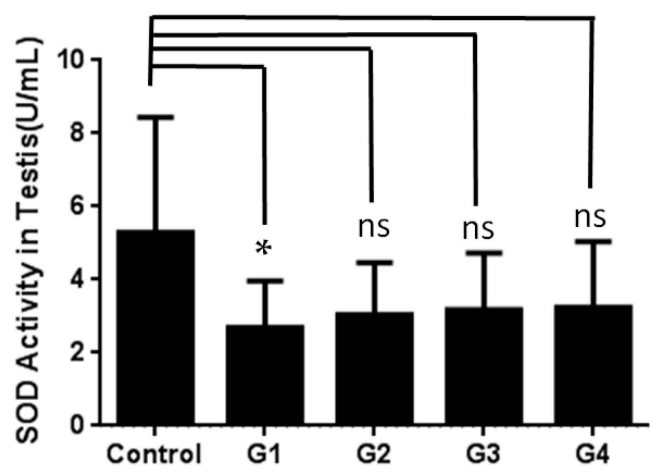

B.

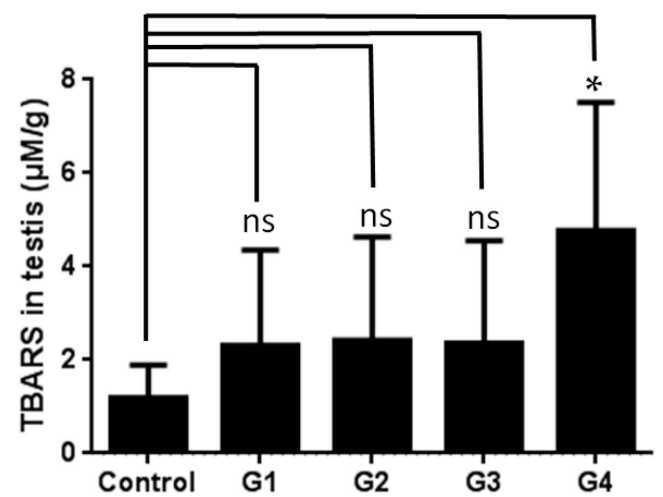

Figure 2. Effects of superoxide dismutase (SOD) activity and thiobarbituric acid reactive substances (TBARS) in testis from mice treated with D-Gal. (A) Mice injected with D-Gal 1 showed decreased SOD activity in testis compared to those administered PBS; (B) mice injected with D-Gal showed increased TBARS activity in testis (TBARS is a type of lipid peroxidation) compared to those administered PBS. One-way ANOVA test: bars represent the mean \pm SD; Student's $t$-test: "ns" denotes nonsignificant; * $p<0.05$.

\subsection{Sperm Quality Decreased in D-Gal-Injected Mice}

A recent study revealed that testis weight and sperm count were affected in D-gal-injected mice [19]. However, detailed information regarding the effects of D-gal treatment on semen parameters remains lacking. In the current study, the D-gal-injected groups exhibited decreased testis weights compared to the control group (Figure 3A,B). Sperm counts in the D-gal-injected groups demonstrated a decreasing trend compared to those of the control group (Figure 3C). Furthermore, all four experimental groups exhibited a statistically-significant increase in immotile sperm compared to the control group (Figure 3D). All four groups of the D-gal-injected mice also exhibited a statistically-significant increase in abnormal sperm morphology compared to the control group (Figure 4). These results indicated that D-gal treatment induced male reproductive aging. 
A.

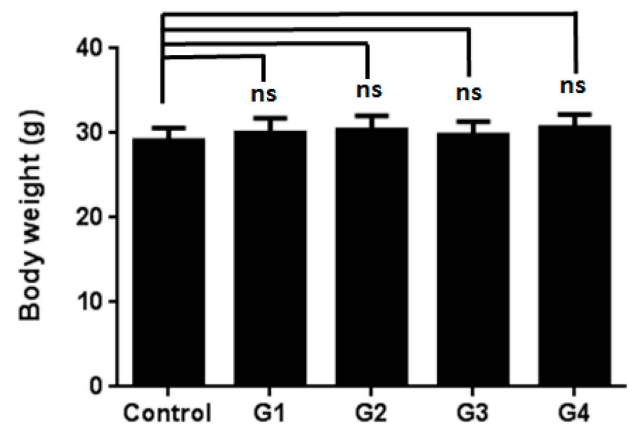

B.

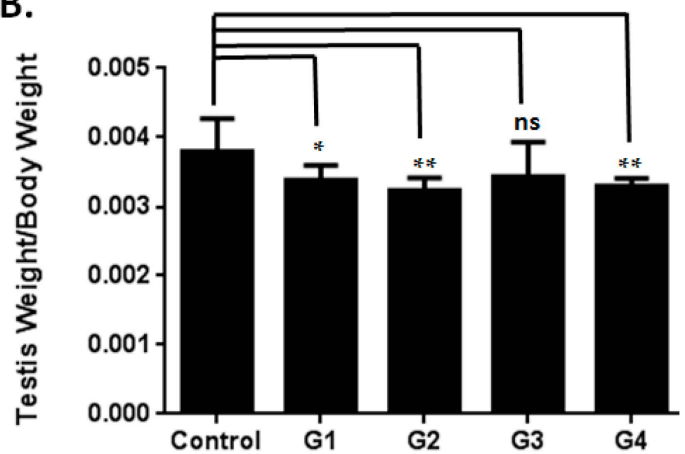

c.

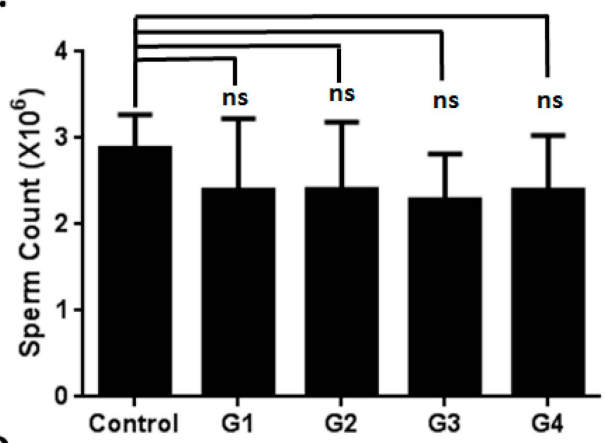

D.

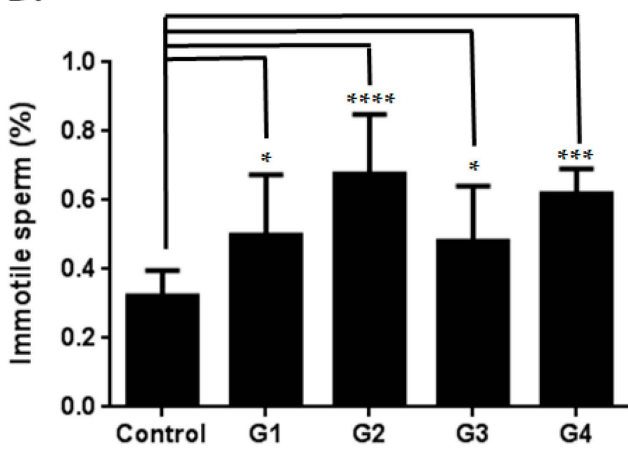

Figure 3. Effects of D-Gal injection on mice (I). (A) Body weights from the different groups are similar; (B) testis weights/body weights from the D-Gal-injected groups show decreases compared to those of the control group; (C) D-Gal-injected groups showed a trend of decreasing sperm counts $\left(\times 10^{6}\right)$ compared to the control group; (D) the D-Gal-injected mice exhibited an increase in immotile sperm (\%) compared to those administered PBS. One-way ANOVA test: bars represent the mean \pm SD; "ns" denotes nonsignificant; ${ }^{*} p<0.05$; ${ }^{* *} p<0.01$; ${ }^{* * *} p<0.001$; ${ }^{* * *} p<0.0001$.

A.

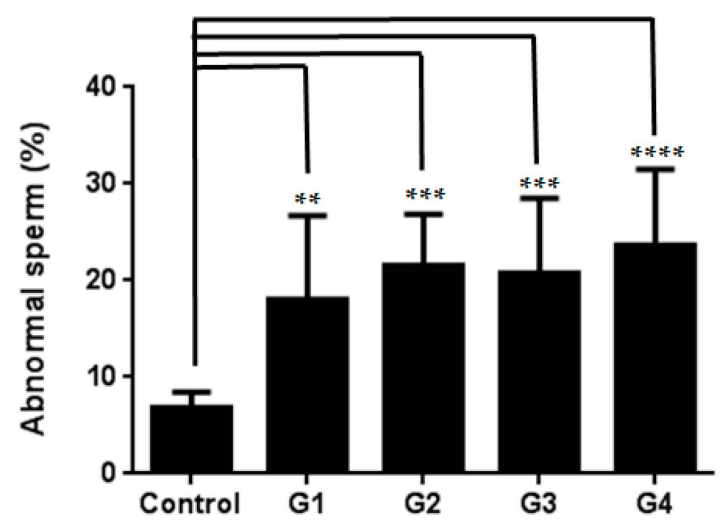

B.

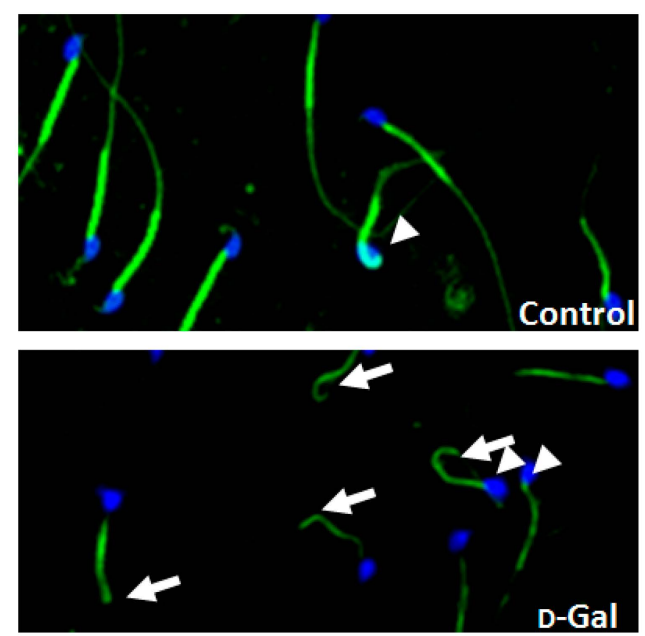

Figure 4. Effects of D-Gal injection on mice (II) (A) D-Gal-injected mice showed increased abnormal sperm morphology (\%) compared to the control group. One-way ANOVA test: bars represent the mean $\pm \mathrm{SD} ;{ }^{* *} p<0.01 ;{ }^{* * *} p<0.001 ;{ }^{* * *} p<0.0001$; (B) Increased abnormal sperm morphology (\%) (arrow: sperm with tail defects; arrowhead: head defects) from mice injected with D-Gal (bottom) compared to those administered PBS. 4,6-diamidino-2-phenylindole (DAPI): blue; Mito Tracker: green (magnification: $\times 400$ ). 


\subsection{Investigating the Possible Mechanism of Reproductive Aging in D-Gal-Injected Mice}

To identify the affected testicular tissue genes in the D-gal-injected mice, c-DNA microarrays were conducted for a global analysis of gene expression. The microarray analyses indicated that, compared to the control group, the D-gal-injected mice significantly exhibited 129 upregulated genes and 20 downregulated genes (Table S1). We selected Glutathione peroxidase 3 (GPx3), the most upregulated gene, for further evaluation. The total amount of GP $x 3$ in the D-gal-injected mice was lower than that in the controls (Figure S1). Nine spermatogenesis-related genes were observed (Table 1). Among the spermatogenesis-related genes, eight genes were upregulated (i.e., Cycl2, HK1, Pltp, Utp3, Cabyr, Zpbp2, Sperr 2 and Csnka2ip) and one (i.e., Katnb1) was downregulated (Table 1). Several of these genes are critical for forming sperm-head morphologies or maintaining nuclear integration (e.g., cylicin, the basic protein of sperm head cytoskeleton 2 (Cylc2); casein kinase 2, an alpha prime interacting protein (Csnka2ip); and katanin p80 (WD40 repeat-containing) subunit B1 (Katnb1). On the basis of these results, we optimized a model of male reproductive aging and determined the effects of D-gal injection on testicular tissue genes.

Table 1. Expressional changes in the spermatogenesis-related genes of the D-gal-injected mice.

\begin{tabular}{ccc}
\hline Gene & Full Name & Fold \\
\hline Cylc2 & Cylicin, basic protein of sperm head cytoskeleton 2 & 5.62 \\
Hk1 & Hexokinase 1 & 2.40 \\
Pltp & Phospholipid transfer protein & 2.25 \\
Utp3 & UTP3, small subunit (SSU) processome component & 2.20 \\
Cabyr & Calcium-binding tyrosine-(Y)-phosphorylation regulated & 2.19 \\
Zpbp2 & Zona pellucida binding protein 2 & 2.13 \\
Speer2 & Spermatogenesis associated glutamate (E)-rich protein 2 & 2.03 \\
Csnka2ip & Casein kinase 2, alpha prime interacting protein & 2.06 \\
Katnb1 & Katanin p80 (WD40-containing) subunit B1 & 0.48 \\
\hline
\end{tabular}

\section{Materials and Methods}

\subsection{Animal Preparation and Experimental Design}

All of the study protocols were executed according to the Guiding Principles for Care and Use of Laboratory Animals and were approved by the Institutional Animal Care and Use Committee (IACUC) of Fu-Jen Catholic University (Identification Code: A10122; 31 July 2012). After an initial acclimatization of 1 week, C57Bl/ 6 mice were randomized into five groups (each containing 8-10 mice) according to the daily intraperitoneal injection of PBS or 100 or $200 \mathrm{mg} / \mathrm{kg}$ dosages of D-gal for 6 or 8 weeks (Figure 1A). The mice were sacrificed $24 \mathrm{~h}$ after the final injection under deep anesthesia, and samples (e.g., blood, testes and sperm from the vas deferens) were collected.

\subsection{Evaluation of Superoxide Dismutase Activity}

Superoxide dismutases (SODs) are critical metalloenzymes that catalyze a superoxide anion to $\mathrm{H}_{2} \mathrm{O}_{2}$ [26]. Blood (0.5 mL) and testicular tissue from the mice were injected with vehicle or D-gal. For the serum samples, the blood collected from the treated mice was allowed to clot for $30 \mathrm{~min}$ at $25^{\circ} \mathrm{C}$ and centrifuged at $2000 \times g$ for $15 \mathrm{~min}$ at $4{ }^{\circ} \mathrm{C}$. Subsequently, the white buffy layer was isolated for further study. For the testis samples, the testes isolated from the mice were homogenized in HEPES (4-(2-hydroxyethyl)-1-piperazineethanesulfonic acid) buffer and centrifuged at $1500 \times g$ for $5 \mathrm{~min}$ at $4{ }^{\circ} \mathrm{C}$. Subsequently, the supernatant was removed. The assay protocol was executed according to the Superoxide Dismutase Assay Kit handbook (No. 706002; Cayman Chemical, Ann Arbor, MI, USA). The final results are presented as $\mathrm{U} / \mathrm{mg}$ proteins. 


\subsection{Thiobarbituric Acid Reactive Substances (TBARS) Analysis}

MDA is a natural product of lipid peroxidation [27]. The MDA level in the testicular tissue was estimated using the TBARS assay [27]. The assay protocol was executed according to the TBARS Assay Kit handbook (No. 10009055; Cayman Chemical, Ann Arbor, MI, USA). The final results are presented as $\mu$ mole MDA/g proteins.

\subsection{Semen Parameter}

Spermatozoa were collected from the vas deferens of the mice and suspended in a human tubal fluid (HTF) medium (Irvine Scientific, Santa Ana, CA, USA). Regarding sperm counts, sperm cells were immobilized by dilution in water and counted in duplicate by using a hemocytometer (Sigma-Aldrich, Saint Louis, MO, USA). For determining the percent motility, the sperm medium was diluted to $10^{6} / \mathrm{mL}$ by using HTF and spotted onto a glass slide. A total of 200 sperm cells (both motile and immotile) were counted in duplicate under a microscope to derive an average percent motility. To analyze the sperm morphology, 100 sperm cells were evaluated on average. Individual sperm cells were categorized as having normal or abnormal morphology (including head, neck and tail defects and immaturity) according to the World Health Organization criteria [3]. The midpiece of each sperm was stained using Mito Tracker conjugated with Alexa Fluor $488(10 \mathrm{mg} / \mathrm{mL})$ (Invitrogen, Carlsbad, CA, USA); 4,6-diamidino-2-phenylindole (DAPI) was used for nuclear staining.

\subsection{Microarray Analysis}

The murine testicular tissue samples were stored in liquid nitrogen until use by using 2-methylbutane as a cryoprotectant. The total cellular RNA was extracted from the biopsies by using the RNAasey Mini Kit (QIAGEN, Venlo, The Netherlands) according to the manufacturer's protocol. Total RNA was quantified by measuring the total absorbance at $\mathrm{OD}_{260} / \mathrm{OD}_{280} \mathrm{~nm}$. The quality of total RNA was monitored using an Agilent 2100 Bioanalyzer (Agilent Technologies, Waldbronn, Germany). Mouse OneArray ${ }^{\circledR}$ v2 (PhalanxBio, Hsinchu, Taiwan) was used in this assay.

\section{Conclusions}

In this study, we optimized a model of male reproductive aging through D-gal injection and examined its suitability for investigating male reproductive aging. In addition, by conducting cDNA microarray analyses, we identified several spermatogenic genes affected by reproductive aging. These results reveal the possible mechanisms of male reproductive aging.

\subsection{Effect of D-Gal Injection on the Expression Levels of Spermatogenesis-Related Genes in Mice}

In this study, we observed a decreased sperm count and increased ratio of immotile and abnormal sperm morphology. In addition, the microarray analyses reveal that nine spermatogenesis-related genes were affected by D-gal injection. Unexpectedly, the upregulation of the Cycl 2 transcripts of the D-gal-injected mice was five-fold higher than that of the controls. Cycl2/CYCL2 is specifically expressed in the testes and is a part of the basic protein of mammalian sperm heads [28,29]. Moreover, CYCL2 plays a role in the morphogenesis of sperm heads. The Katnb1 transcripts of the D-gal-injected mice decreased two-fold compared to those of the controls. Katanin is a heterodimer comprising a 60-kDa ATPase subunit A 1 (Katna1) and an 80-kDa accessory protein subunit B 1 (Katnb1) [30]. The p60 subunit disassembles microtubules, and the p80 subunit targets katanin to centrosomes. Katnb1 is presented during spermatid development and is particularly localized in the microtubules of the manchette, a structure required for sperm-head shaping [31]. Mutation in Katnb1 results in male sterility, which is characterized by decreased sperm count and an absence of progressive sperm in addition to abnormal sperm-head morphology. Genetic alternations of Katnb1 were observed in infertile men with oligoasthenoteratozoospermia [32]. The spermatogenic genes affected by D-gal 
injection may reflect the mechanism of spermatogenesis; this phenomenon is similar to the aging process during spermatogenesis.

\subsection{Model of Male Reproductive Aging}

A previous study reported that D-gal forms advanced glycation end products (AGEs) in vivo and that elevated levels of AGEs may accelerate the aging process [20]. In our study, the D-gal-injected mice demonstrated decreased SOD activity and increased lipid peroxidation levels. These results are consistent with those of previous studies that have applied D-gal to induce brain aging in mice (Figure 5) [23,25,33]. Low SOD activity reflects normal male aging [34]. Previous studies involving D-gal-injected rats have reported that the rats exhibited a decrease in sperm count and a regression of the testes, which is consistent with our results [34]. However, only one study that employed D-gal-injected mice for evaluating male reproductive aging revealed decreased sperm counts and increased serum LH levels [19]; our results, which showed decreased testis weights and lower sperm counts, are consistent with the results of this study. Furthermore, we found an increased ratio of immotile sperm and abnormal sperm morphology in the D-gal-treated mice (Figure 5); this finding is highly consistent with the typical characteristics of aging males (e.g., reduced total sperm count, concentration and motility; increased abnormal sperm morphology) [4,5]. On the basis of these results, we conclude that the optimized model of reproductive aging involving D-gal-injected mice is suitable for investigating male reproductive aging. Although previous studies have recommended using antioxidants for treating infertility involving ROS in males, the reports of studies on the therapeutic effects of such antioxidants have been contradictory $[13,15,16]$. Therefore, we optimized this model for evaluating the effects of antioxidants on male reproductive aging.

Testis: D-Galactose injection (induced ROS accumulation)

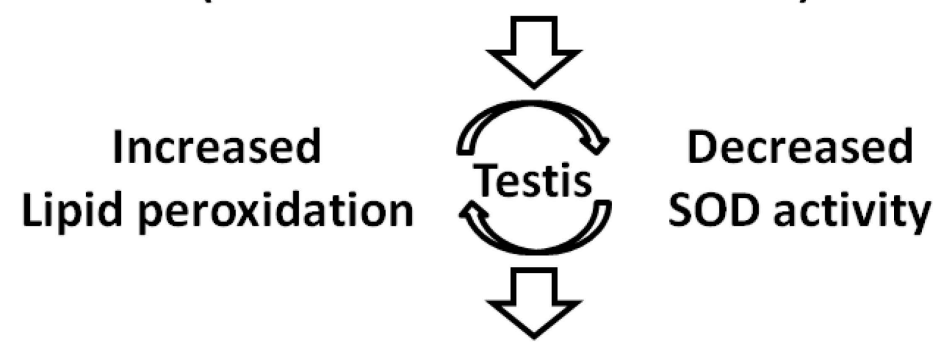

Affected the spermatogenic related genes

(e.g., Cycl2 and Katnb1)

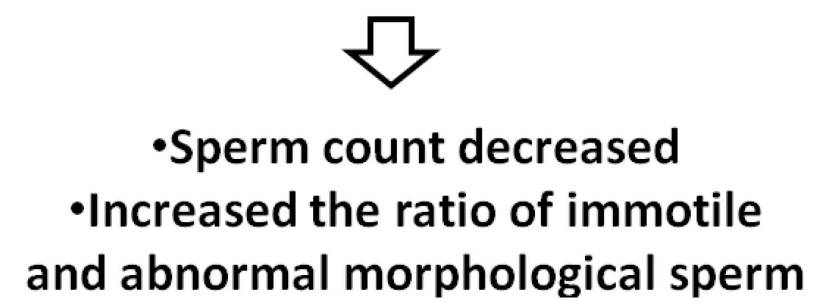

Figure 5. Mouse model of male reproductive aging induced by D-Galactose injection. The D-Gal-injected mice demonstrated decreased SOD activity and increased lipid peroxidation amount. Further, SOD accumulation affects the expression of spermatogenic genes (e.g., Cycl2 and Katnb1) and results in the decreased sperm count and increased ratio of immotile and abnormal sperm morphology. 


\subsection{Conclusions}

D-gal injection induced male reproductive aging and may affect several spermatogenic genes. D-gal-treated mice may be excellent candidates for demonstrating the aging of the male reproductive system.

Supplementary Materials: Supplementary materials can be found at http://www.mdpi.com/1422-0067/ $17 / 1 / 98 / \mathrm{s} 1$.

Acknowledgments: This study was supported by grants from the Ministry of Science and Technology of the Republic of China (Ying-Hung Lin: National Science Council (NSC) 101-2313-B-030-006- and NSC 102-2320-B-030-006-MY3) and from Cardinal Tien Hospital (Ying-Hung Lin: CTH-102-1-2B10).

Author Contributions: Chun-Hou Liao: Designed the study and wrote the manuscript; Bing-Huei Chen: Designed the study, provided reagents and conceptual advice; Han-Sun Chiang: Gave technical support, provided reagents and conceptual advice; Chiu-Wei Chen: Performed experiments, designed the study, and analyzed data; Mei-Feng Chen: Gave technical support and conceptual advice; Chih-Chun Ke: Conceptual advice and edited the manuscript; Ya-Yun Wang: Performed experiments and designed the study; Wei-Ning Lin: Gave technical support and conceptual advice; Chi-Chung Wang: Gave technical support and conceptual advice; Ying-Hung Lin: Designed the study and wrote the manuscript.

Conflicts of Interest: The authors declare no conflict of interest.

\section{References}

1. Charlton, B.G. Psychological neoteny and higher education: Associations with delayed parenthood. Med. Hypotheses 2007, 69, 237-240. [CrossRef] [PubMed]

2. Kovac, J.R.; Addai, J.; Smith, R.P.; Coward, R.M.; Lamb, D.J.; Lipshultz, L.I. The effects of advanced paternal age on fertility. Asian J. Androl. 2013, 15, 723-728. [CrossRef] [PubMed]

3. World Health Organization (WHO). WHO Laboratory Manual for the Examination of Human Semen and Sperm-Cervical Mucus Interaction; Cambridge University Press: Cambridge, UK, 1999.

4. Mukhopadhyay, D.; Varghese, A.C.; Pal, M.; Banerjee, S.K.; Bhattacharyya, A.K.; Sharma, R.K.; Agarwal, A. Semen quality and age-specific changes: A study between two decades on 3729 male partners of couples with normal sperm count and attending an andrology laboratory for infertility-related problems in an Indian city. Fertil. Steril. 2010, 93, 2247-2254. [CrossRef] [PubMed]

5. Wyrobek, A.J.; Eskenazi, B.; Young, S.; Arnheim, N.; Tiemann-Boege, I.; Jabs, E.W.; Glaser, R.L.; Pearson, F.S.; Evenson, D. Advancing age has differential effects on DNA damage, chromatin integrity, gene mutations, and aneuploidies in sperm. Proc. Natl. Acad. Sci. USA 2006, 103, 9601-9606. [CrossRef] [PubMed]

6. Cocuzza, M.; Athayde, K.S.; Agarwal, A.; Sharma, R.; Pagani, R.; Lucon, A.M.; Srougi, M.; Hallak, J. Age-related increase of reactive oxygen species in neat semen in healthy fertile men. Urology 2008, 71, 490-494. [CrossRef] [PubMed]

7. Desai, N.; Sabanegh, E., Jr.; Kim, T.; Agarwal, A. Free radical theory of aging: Implications in male infertility. Urology 2010, 75, 14-19. [CrossRef] [PubMed]

8. Raha, S.; Robinson, B.H. Mitochondria, oxygen free radicals, disease and ageing. Trends Biochem. Sci. 2000, 25, 502-508. [CrossRef]

9. Muller, F.L.; Lustgarten, M.S.; Jang, Y.; Richardson, A.; van Remmen, H. Trends in oxidative aging theories. Free Radic. Biol. Med. 2007, 43, 477-503. [CrossRef] [PubMed]

10. Beckman, K.B.; Ames, B.N. The free radical theory of aging matures. Physiol. Rev. 1998, 78, 547-581. [PubMed]

11. D'Agata, R.; Vicari, E.; Moncada, M.L.; Sidoti, G.; Calogero, A.E.; Fornito, M.C.; Minacapilli, G.; Mongioi, A.; Polosa, P. Generation of reactive oxygen species in subgroups of infertile men. Int. J. Androl. 1990, 13, 344-351. [CrossRef] [PubMed]

12. Sharma, R.K.; Agarwal, A. Role of reactive oxygen species in male infertility. Urology 1996, 48, 835-850. [CrossRef]

13. Ross, C.; Morriss, A.; Khairy, M.; Khalaf, Y.; Braude, P.; Coomarasamy, A.; El-Toukhy, T. A systematic review of the effect of oral antioxidants on male infertility. Reprod. Biomed. Online 2010, 20, 711-723. [CrossRef] [PubMed]

14. Blahak, P. Vitamin E in the treatment of male sterility. Ceskoslovenska Gynekol. 1947, 12, 80-85. 
15. Abel, B.J.; Carswell, G.; Elton, R.; Hargreave, T.B.; Kyle, K.; Orr, S.; Rogers, A.; Baxby, K.; Yates, A. Randomised trial of clomiphene citrate treatment and vitamin $C$ for male infertility. Br. J. Urol. 1982, 54, 780-784. [CrossRef] [PubMed]

16. Hargreave, T.B.; Kyle, K.F.; Baxby, K.; Rogers, A.C.; Scott, R.; Tolley, D.A.; Abel, B.J.; Orr, P.S.; Elton, R.A. Randomised trial of mesterolone versus vitamin C for male infertility. Scottish Infertility Group. Br. J. Urol. 1984, 56, 740-744. [CrossRef] [PubMed]

17. Bruni, J.F.; Huang, H.H.; Marshall, S.; Meites, J. Effects of single and multiple injections of synthetic GnRH on serum LH, FSH and testosterone in young and old male rats. Biol. Reprod. 1977, 17, 309-312. [CrossRef] [PubMed]

18. Bethea, C.L.; Walker, R.F. Age-related changes in reproductive hormones and in Leydig cell responsivity in the male Fischer 344 rat. J. Gerontol. 1979, 34, 21-27. [CrossRef] [PubMed]

19. Ahangarpour, A.; Oroojan, A.A.; Heidari, H. Effects of exendin-4 on male reproductive parameters of D-galactose induced aging mouse model. World J. Mens Health 2014, 32, 176-183. [CrossRef] [PubMed]

20. Song, X.; Bao, M.; Li, D.; Li, Y.M. Advanced glycation in D-galactose induced mouse aging model. Mech. Ageing Dev. 1999, 108, 239-251. [CrossRef]

21. Pirke, K.M.; Vogt, H.J.; Geiss, M. In vitro and in vivo studies on Leydig cell function in old rats. Acta Endocrinol. 1978, 89, 393-403. [CrossRef] [PubMed]

22. Paul, C.; Nagano, M.; Robaire, B. Aging results in differential regulation of DNA repair pathways in pachytene spermatocytes in the Brown Norway rat. Biol. Reprod. 2011, 85, 1269-1278. [CrossRef] [PubMed]

23. Ho, S.C.; Liu, J.H.; Wu, R.Y. Establishment of the mimetic aging effect in mice caused by D-galactose. Biogerontology 2003, 4, 15-18. [CrossRef] [PubMed]

24. Bucala, R.; Cerami, A. Advanced glycosylation: Chemistry, biology, and implications for diabetes and aging. Adv. Pharmacol. 1992, 23, 1-34. [PubMed]

25. Lu, J.; Zheng, Y.L.; Wu, D.M.; Luo, L.; Sun, D.X.; Shan, Q. Ursolic acid ameliorates cognition deficits and attenuates oxidative damage in the brain of senescent mice induced by D-galactose. Biochem. Pharmacol. 2007, 74, 1078-1090. [CrossRef] [PubMed]

26. Novak, R.; Bosze, Z.; Matkovics, B.; Fachet, J. Gene affecting superoxide dismutase activity linked to the histocompatibility complex in H-2 congenic mice. Science 1980, 207, 86-87. [CrossRef] [PubMed]

27. Armstrong, D.; Browne, R. The analysis of free radicals, lipid peroxides, antioxidant enzymes and compounds related to oxidative stress as applied to the clinical chemistry laboratory. Adv. Exp. Med. Biol. 1994, 366, 43-58. [PubMed]

28. Von Bulow, M.; Heid, H.; Hess, H.; Franke, W.W. Molecular nature of calicin, a major basic protein of the mammalian sperm head cytoskeleton. Exp. Cell Res. 1995, 219, 407-413. [CrossRef] [PubMed]

29. Hess, H.; Heid, H.; Zimbelmann, R.; Franke, W.W. The protein complexity of the cytoskeleton of bovine and human sperm heads: the identification and characterization of cylicin II. Exp. Cell Res. 1995, 218, 174-182. [CrossRef] [PubMed]

30. Hartman, J.J.; Mahr, J.; McNally, K.; Okawa, K.; Iwamatsu, A.; Thomas, S.; Cheesman, S.; Heuser, J.; Vale, R.D.; McNally, F.J. Katanin, a microtubule-severing protein, is a novel AAA ATPase that targets to the centrosome using a WD40-containing subunit. Cell 1998, 93, 277-287. [CrossRef]

31. O’Donnell, L.; Rhodes, D.; Smith, S.J.; Merriner, D.J.; Clark, B.J.; Borg, C.; Whittle, B.; O'Connor, A.E.; Smith, L.B.; McNally, F.J.; et al. An essential role for katanin p80 and microtubule severing in male gamete production. PLoS Genet. 2012, 8. [CrossRef] [PubMed]

32. O’Donnell, L.; McLachlan, R.I.; Merriner, D.J.; O’Bryan, M.K.; Jamsai, D. KATNB1 in the human testis and its genetic variants in fertile and oligoasthenoteratozoospermic infertile men. Andrology 2014, 2, 884-891. [CrossRef] [PubMed]

33. Tsai, S.J.; Yin, M.C. Anti-oxidative, anti-glycative and anti-apoptotic effects of oleanolic acid in brain of mice treated by D-galactose. Eur. J. Pharmacol. 2012, 689, 81-88. [CrossRef] [PubMed]

34. Luo, L.; Chen, H.; Trush, M.A.; Show, M.D.; Anway, M.D.; Zirkin, B.R. Aging and the brown Norway rat leydig cell antioxidant defense system. J. Androl. 2006, 27, 240-247. [CrossRef] [PubMed]

(C) 2016 by the authors; licensee MDPI, Basel, Switzerland. This article is an open access article distributed under the terms and conditions of the Creative Commons by Attribution (CC-BY) license (http://creativecommons.org/licenses/by/4.0/). 